\title{
EL EFECTO TRASPASO DE LA TASA DE INTERÉS DE LOS INSTRUMENTOS DEL BANCO CENTRAL EN COSTA RICA HACIA LAS TASAS DE INTERÉS DEL SISTEMA FINANCIERO
}

\author{
José Pablo Barquero Romero ${ }^{1}$ \\ David Ricardo Mora Guerrero ${ }^{2}$
}

Recibido: 01/08/2014

Aprobado: 26/06/2015

\begin{abstract}
RESUMEN
El trabajo consta de dos partes. En la primera se prueban, a partir del enfoque de cointegración de Engle y Granger (1987), las siguientes hipótesis: i) el efecto traspaso es unitario, ii) el efecto traspaso sufrió una variación al adoptar un nuevo arreglo cambiario en octubre de 2006, iii) la velocidad de ajuste aumentó con el cambio del régimen cambiario (no linealidad), iv) los bancos del sistema financiero no ajustan simétricamente las tasas de interés (con el fin de aumentar su margen de intermediación). En la segunda parte, se analizan los factores que influyen sobre la efectividad del mecanismo de transmisión, entre ellos, la dolarización financiera, la concentración bancaria, el nivel de deuda interna del Gobierno y el déficit primario del Banco Central de Costa Rica. Estas últimas dos variables, como porcentaje del Producto Interno Bruto (PIB).
\end{abstract}

PALABRAS CLAVE: EFECTO TRASPASO, TASA DE POLÍTICA MONETARIA, ASIMETRÍA, NO LINEALIDAD, DOLARIZACIÓN, DEUDA INTERNA,CONCENTRACIÓN BANCARIA.

\section{ABSTRACT}

The paper has two parts, the first part tests from the Engle and Granger (1987) point of view the following hypothesis: i) the pass through is equal to one, ii) the pass through changed after the adoption of a new exchange rate regime, iii) the pass through speed changed along with the change in the exchange rate regime (non-linearity), iv) the banks in the financial system do not adjust symmetrically their interest rates (in order to increase their profit margin). In the second part the paper analyses the main factors that affect the effectiveness of the pass through mechanism, financial dollarization, banking industry concentration, the level of government debt and the Central Bank deficit.

KEYWORDS: PASS-THROUGH, POLICY-RATE, ASYMMETRIC, NON-LINEARITY, DOLLARIZATION, DEBT, BANK CONCENTRATION.

1 Banco Central de Costa Rica, Departamento de Investigación Económica, Código Postal 10058-1000, Costa Rica; barquerorj@bccr.fi.cr

2 Universidad Nacional de Costa Rica, estudiante Programa de Licenciatura en Economía,Heredia, Costa Rica, Código postal: 86-3000; davidricardo0990@hotmail.com 


\section{INTRODUCCIÓN}

El Banco Central de Costa Rica (BCCR) estableció la tasa de política monetaria (TPM) como su principal instrumento de política monetaria en junio del 2011. Esto como uno de varios pasos hacia la adopción de un régimen monetario de Meta de Inflación (MI). Sin embargo, con el fin de realizar comparaciones en el tiempo, este trabajo recurre a un indicador alternativo a la TPM, que refleje el tono de la política monetaria (ITPM) para el periodo en el cual no se dispone de una TPM ${ }^{3}$ explícita. Por lo tanto, este estudio se enfoca en explorar con los datos disponibles cómo opera el mecanismo, a partir del cual un cambio en la tasa de los instrumentos de política monetaria puede influir las otras tasas del sistema financiero, para que luego esto incida en las decisiones de consumo e inversión, así como en la demanda agregada y en la inflación.

El presente estudio contiene dos tipos de análisis. El primero utiliza el enfoque de cointegración de Engle-Granger (1987), con el cual se prueban las hipótesis de traspaso unitario, no linealidad y asimetría del efecto traspaso. El segundo análisis, siguiendo a Medina et al. (2011), examina factores que interrumpen el traspaso de la tasa de política hacia las demás tasas del sistema financiero costarricense. Los efectos por considerar corresponden al nivel de dolarización de los depósitos, la concentración bancaria medida por el índice de Herfindahl-Hirschman, el saldo primario del Gobierno Central y el déficit del BCCR, estos dos últimos como porcentaje del Producto Interno Bruto (PIB).

Esta investigación abarca el periodo de enero de 1999 a julio de 2013. Para fines de análisis, este periodo se dividirá en: antes de octubre del 2006 y después de ese mes. Esto ya que en esta fecha, el BCCR adopta un sistema cambiario de banda. Esta es una acción dirigida a dar un mayor grado de libertad al BCCR para manejar su política monetaria, es decir "romper" la Trinidad Imposible 4 . Adicionalmente, se utilizan siete tasas de interés: cuatro pasivas y tres activas, representativas de las tasas de interés que componen el sistema financiero, las cuales se describirán con más detalle en las secciones siguientes.

Para este estudio y desde el punto de vista teórico, el efecto traspaso ocurre cuando el Banco Central varía la tasa de interés de sus instrumentos y esta variación se traslada al resto de tasas del sistema financiero. Los trabajos más recientes en Costa Rica sobre este tema son los realizados por Durán y Esquivel (2008) y por Monge y Muñoz (2011); sin embargo, estos estudios no abarcan el periodo en el que BCCR contó con una tasa de política monetaria como tasa de referencia y, por tanto, los resultados del presente estudio son de particular importancia para las autoridades monetarias costarricenses. Adicionalmente, como se mencionó, aun cuando en periodos previos al año 2011 el BCCR no contaba con una tasa de política monetaria explicita como la actual, el ente emisor sí utilizaba sus instrumentos de cortos plazos para enviar sus señales al mercado.

El primero encuentra evidencia estadística de que el traspaso de cambios en la tasa de política monetaria hacia las tasas de interés del sistema financiero es completo, tanto para las tasas activas como para las pasivas. Además, según afirman los autores, existe una tendencia de un traslado mayor hacia los bancos privados en contraste con los estatales. Concluyen mencionando que el efecto traspaso sufrió una variación tras adoptar el régimen de banda cambiaria (no linealidad), y no encuentran evidencia de asimetría en el traspaso.

Por su parte, Monge y Muñoz (2011) señalan que no siempre el traspaso es completo y que tarda una cantidad determinada de tiempo en trasladarse al resto de tasas del mercado. Enumeran como razones las siguientes: las características estructurales de la industria -si es perfec-

3 Desde junio del 2011 y hasta diciembre del 2013, la TPM se ha ajustado solamente en dos ocasiones.

4 Basada en el modelo de Mundell-Fleming, propone que es imposible para una economía abierta tener al mismo tiempo un tipo de cambio fijo, libre movilidad de capitales y una política monetaria autónoma.

Ciencias Económicas 33-N¹: 2015/ 37-61 / ISSN: 0252-9521 
tamente competitiva o pocos agentes concentran un gran poder de mercado-; la existencia de información imperfecta y otras distorsiones de mercado. En su criterio, esto puede generar rigideces que afectan la velocidad y la magnitud con que reacciona el sistema financiero. Así, una alta concentración bancaria limita la competencia y reduce la reacción de los bancos ante la tasa de política $y$, por consiguiente, pueden disminuir la magnitud y la velocidad del mecanismo de transmisión hacia las demás tasas de interés.

Monge y Muñoz (2011) observan, además, que en el periodo de enero de 2000 a noviembre de 2010, en promedio, las tasas de interés activas poseen un coeficiente de traspaso de largo plazo mayor en comparación con las tasas de interés pasivas. También concluyen que existe evidencia de un cambio estructural a partir de la adopción del nuevo régimen cambiario en octubre de 2006. Por último, encuentran que la velocidad de los movimientos de traspaso de la tasa de política monetaria es mayor en las tasas activas con respecto a las pasivas (ocho y trece meses, respectivamente).

Medina et al. (2011) estudian el efecto traspaso en los países centroamericanos y determinan que este efecto es más débil y lento en Centroamérica en relación con los países de la LA6 ${ }^{5}$. Este estudio incluyó a Costa Rica dentro de su marco de estudio y difiere con el estudio propuesto en este documento en el sentido que los autores utilizaron datos anuales y, como tasa de interés de política, emplean la Tasa Básica Pasiva (TBP); mientras que este estudio propone mejorar el análisis al utilizar datos mensuales y un indicador de la tasa de política monetaria (ITPM).

Medina et al. (2011) también estudian qué otros factores pueden influir en el efecto traspaso y citan los siguientes:

i. Alto grado de dolarización financiera, que puede limitar la dinámica del efecto traspaso por cuanto los agentes, ante variaciones en las tasas, pueden cambiar hacia instrumentos en moneda extranjera (efecto de sustitución), lo cual termina limitando el margen de acción de la política monetaria.

ii. Dominancia fiscal, por ejemplo, los préstamos del Banco Central hacia el Gobierno afectan el balance del Banco Central y limitan la capacidad de este para brindar señales claras acerca de hacia dónde va orientada la política monetaria.

iii. Altos déficits fiscales o altos niveles de deuda interna, los cuales pueden elevar las tasas de interés e interferir con el efecto traspaso.

iv. Mercados financieros poco competitivos.

Adicionalmente, señalan que tener un tipo de cambio fluctuante posibilita que la tasa de interés de política del Banco Central, instrumento primario de política monetaria, envíe señales más claras para los participantes del mercado, además de aumentar la independencia de la política monetaria. Por último, la literatura señala que existen otros factores como la estabilidad y el tamaño del sistema financiero, la credibilidad del Banco Central, la calidad del marco regulatorio y el entorno institucional que pueden también afectar el traspaso de la tasa de política monetaria hacia las tasas de interés del sistema financiero.

\section{METODOLOGÍA}

Para analizar el efecto traspaso que tiene la tasa de interés de los instrumentos de política monetaria del BCCR sobre las tasas de interés de mercado, se seleccionaron las siguientes variables para el periodo de enero de 1999 a julio de 2013, utilizando promedios mensuales de datos diarios:

5 Los países que componen el grupo denominado LA6 son Brasil, Chile, Colombia, México, Perú y Uruguay. 


\begin{tabular}{ll}
\hline Abreviatura & Variable \\
\hline ITPM & Indicador de tasa de política monetaria \\
TBP & Tasa básica pasiva \\
TA_SF & Tasa de interés activa promedio del sistema financiero \\
TP_SF & Tasa de interés pasiva promedio del sistema financiero \\
TOA_BE & Tasa de interés activa para "otras actividades" de los bancos estatales \\
TOA_BP & Tasa de Interés Activa para "otras actividades" de los bancos privados \\
TD6_BE & Tasa de interés pasiva a seis meses plazo de los bancos estatales \\
TD6_BP & Tasa de interés pasiva a seis meses plazo de los bancos privados \\
\hline
\end{tabular}

El cuadro 1 muestra las estadísticas descriptivas de las variables utilizadas en el análisis para el periodo del estudio.

CUADRO 1

ESTADÍSTICAS DESCRIPTIVAS PARA LAS TASAS DEL SISTEMA FINANCIERO

\begin{tabular}{lcccccccc}
\hline & TA_SF & TBP & TD6_BE & TD6_BP & TOA_BE & TOA_BP & TP_SF & ITPM \\
\hline Promedio & 22,76 & 12,76 & 10,11 & 11,84 & 21,48 & 24,82 & 10,32 & 7,47 \\
Mediana & 23,21 & 13,64 & 10,51 & 13,41 & 22,36 & 24,81 & 11,40 & 7,34 \\
Máximo & 30,50 & 24,50 & 18,07 & 20,44 & 29,51 & 31,81 & 18,74 & 10,60 \\
Mínimo & 14,32 & 4,49 & 4,22 & 3,52 & 12,85 & 15,95 & 3,64 & 3,25 \\
Desv. Est. & 4,21 & 4,53 & 3,24 & 4,22 & 4,57 & 3,70 & 3,97 & 2,04 \\
\hline
\end{tabular}

Con el fin de verificar si existe una relación de precedencia entre las variables, se realizan pruebas de causalidad en el sentido de Granger. Como lo muestra el cuadro 1 del anexo 2, no se puede rechazar la hipótesis nula $y$, por lo tanto, existe evidencia de que los movimientos en la tasa de política monetaria causan, en el sentido de Granger, a los movimientos en las tasas del sistema financiero. También, se encontró que los movimientos en las tasas pasivas preceden a los movimientos en las tasas activas (cuadro 2, anexo 2) y que los movimientos en las tasas activas de los bancos estatales causan, en el sentido de Granger, los movimientos en las tasas activas de los bancos privados (cuadro 3, anexo 2). En el diagrama 1 se aprecia la relación observada en las variables, de acuerdo con estas pruebas, útiles para comprender de forma inicial cómo se comporta el sistema financiero.

6 Dentro de las ventajas del ITPM, se tiene que siempre existe información sobre la tasa, sin tener que recurrir a estimaciones, en contraposición, por ejemplo, de la tasa de los BEM a seis meses plazos que, en ocasiones, no presenta colocaciones en el mercado. Adicionalmente, la serie puede seguir actualizándose en el futuro, porque está en línea con el esquema de control actual del BCCR. El ITPM muestra una correlación con la tasa básica pasiva y con la tasa de los BEM a seis meses plazo, de 0,86 y 0,84, respectivamente (Castro y Chaverri, 2013). Ver anexo 1 para más información.

$7 \quad$ La tasa de interés de "otras actividades" está conformada por consumo, comercio y servicios, cuyos saldos representan el $54 \%$ del total de crédito al sector privado. 


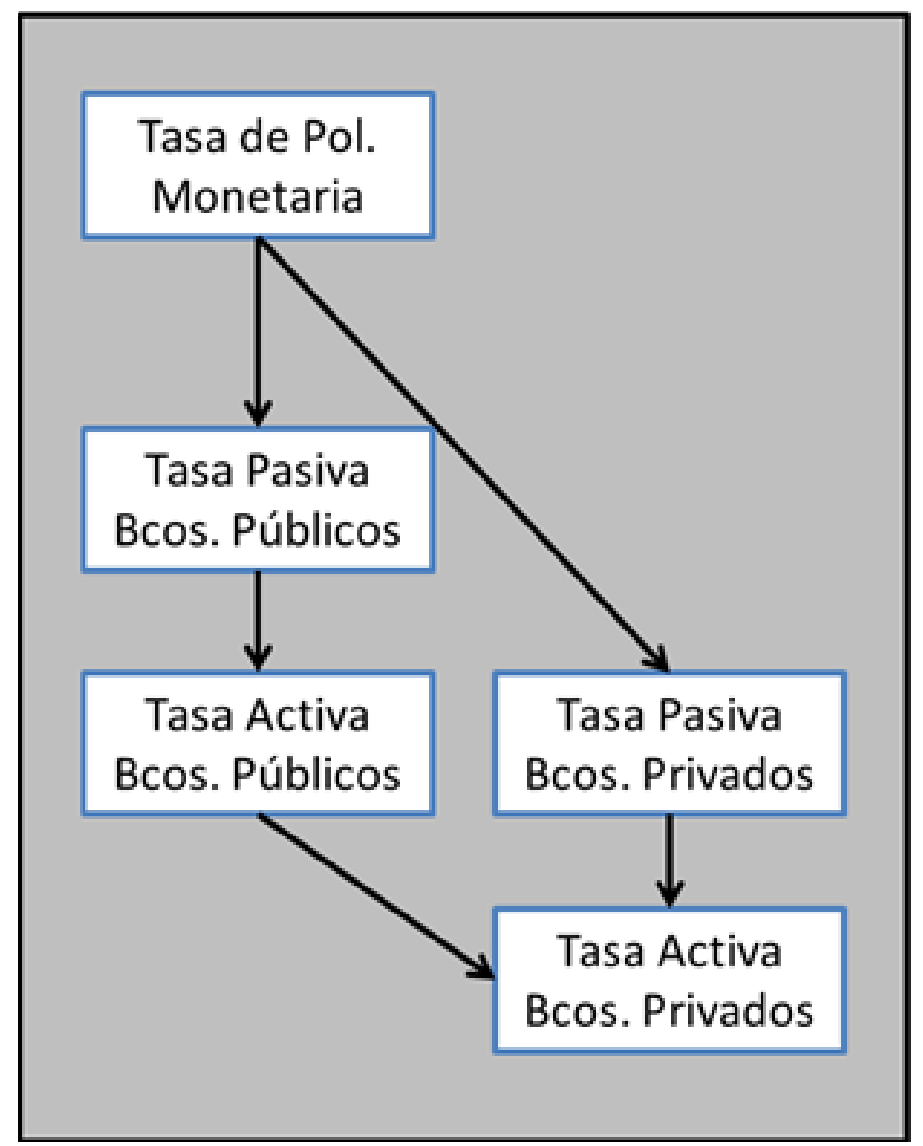

Fuente: Elaboración propia.

A continuación, se verifica la estacionariedad de las series. Para esto, se utiliza la prueba de raíz unitaria de Dickey-Fuller aumentada. El cuadro 1 del anexo 3 contiene el resultado de estas pruebas, de las cuales se concluye que no se puede rechazar la presencia de raíz unitaria de las variables en nivel. Al efectuar de nuevo las pruebas para las series en primera diferencia, se concluye que todas poseen el mismo orden de integración y, por ello, se puede usar el enfoque de cointegración de Engle-Granger.

Seguidamente, se formularon modelos de largo plazo para evaluar los residuos de la regresión y determinar si existe una relación de largo plazo entre las tasas de interés y la tasa de política monetaria. En el caso en que los residuos de la regresión poseen un grado de integración de orden cero, se establece que se obtuvieron a partir de una regresión cointegrante; en caso contrario, los residuos se obtuvieron de una regresión espuria. Los resultados de estas pruebas se resumen en el cuadro 2 del anexo 3; de ellas, se puede concluir que hay evidencia estadística de respaldo a la hipótesis de cointegración entre las variables y la tasa de política monetaria.

Posterior a la estimación de los coeficientes de traspaso, se evalúan las hipótesis de traspaso unitario por medio de los modelos de largo plazo, así como las hipótesis de linealidad y asimetría mediante la utilización de modelos de corrección del error. Los resultados de estas estimaciones se exponen en los apartados siguientes. 


\section{RESULTADOS}

Para estimar el efecto traspaso de largo plazo, se utilizó el periodo de enero de 2000 a julio de 2013. Además, se formularon dos tipos de modelos de largo plazo, el primero sin considerar el cambio en el régimen cambiario del año 2006 y el segundo introduciendo este cambio empleando una variable dicotómica. Los modelos formulados son los siguientes:

Modelo sin modificación del régimen cambiario

$$
\mathrm{R}_{\mathrm{t}}^{\mathrm{i}}=\beta_{\mathrm{i} 1}+\beta_{\mathrm{i} 2} I T P M_{\mathrm{t}}+\varepsilon_{\mathrm{it}}
$$

Modelo con modificación del régimen cambiario

$$
\mathrm{R}_{\mathrm{t}}^{\mathrm{i}}=\beta_{\mathrm{i} 1}+\mathrm{d}_{\mathrm{pa}} \beta_{\mathrm{i} 21} I T P M_{\mathrm{t}}+\mathrm{d}_{\mathrm{bc}} \beta_{\mathrm{i} 22} I T P M_{\mathrm{t}}+\varepsilon_{\mathrm{it}}
$$

En ambos modelos, cada una de las tasas del sistema financiero Ri depende de la tasa de política monetaria $I T P M_{\mathrm{t}}$. En el segundo modelo, se introducen las variables dicotómicas $\mathrm{d}_{\mathrm{pa}} y \mathrm{~d}_{\mathrm{bc}}$, las cuales crean un modelo independiente para cada uno de los dos periodos, anterior y posterior, a la puesta en práctica del régimen de banda cambiaria. Así, $\mathrm{d}_{\mathrm{pa}}$ toma un valor de uno durante el periodo de paridad ajustable $y$ un valor de cero durante el periodo de banda cambiaria $y$ de forma contraria para $\mathrm{d}_{\mathrm{bc}}$.

Es decir, se estiman dos modelos, uno para antes del cambio de régimen y otro para después, en ambos modelos el coeficiente de traspaso es estimado de forma independiente y capturado por el $\beta_{\mathrm{i}}$ respectivo. Luego, se evaluaron las hipótesis de traspaso unitario y de igualdad en coeficientes entre periodos por medio de la prueba de Wald. Los resultados de estas pruebas son los siguientes (cuadro 2):

CUADRO 2

EFECTO TRASPASO DE LA TASA DE POLÍTICA MONETARIA (ITPM) A LAS TASAS DEL SISTEMA

\begin{tabular}{|c|c|c|c|c|c|c|c|}
\hline & \multicolumn{4}{|c|}{ Pasivas } & \multicolumn{3}{|c|}{ Activas } \\
\hline & TBP & TP_SF & TD6_BE & TD6_BP & TA_SF & TOA_BE & TOA_BP \\
\hline \multicolumn{8}{|c|}{ A. Periodo enero de 2000 -julio de 2013} \\
\hline Efecto traspaso & 0,75 & 0,82 & 0,73 & 0,80 & 0,69 & 0,54 & 0,63 \\
\hline Desv. estándar & 0,08 & 0,06 & 0,07 & 0,08 & 0,12 & 0,12 & 0,14 \\
\hline Estadístico $t$ & 8,88 & 12,87 & 11,16 & 10,36 & 6,01 & 4,41 & 4,52 \\
\hline \multicolumn{8}{|c|}{ B. Periodo enero de 2000 -octubre de 2006} \\
\hline Efecto traspaso & 0,56 & 0,61 & 0,55 & 0,50 & 0,51 & 0,37 & 0,52 \\
\hline Desv. estándar & 0,12 & 0,09 & 0,09 & 0,11 & 0,14 & 0,16 & 0,14 \\
\hline Estadístico t & 4,57 & 6,67 & 5,85 & 4,62 & 3,66 & 2,29 & 3,60 \\
\hline \multicolumn{8}{|c|}{ C. Periodo octubre de 2006 -julio de 2013} \\
\hline Efecto traspaso & 0,92 & 1,01 & 0,89 & 1,06 & 1,06 & 0,85 & 1,05 \\
\hline Desv. estándar & 0,11 & 0,08 & 0,09 & 0,10 & 0,13 & 0,15 & 0,14 \\
\hline Estadístico t & 8,01 & 11,89 & 10,07 & 10,43 & 8,02 & 5,54 & 7,60 \\
\hline
\end{tabular}
FINANCIERO 
Continuación...

\begin{tabular}{|c|c|c|c|c|c|c|c|}
\hline & \multicolumn{4}{|c|}{ Pasivas } & \multicolumn{3}{|c|}{ Activas } \\
\hline & TBP & TP_SF & TD6_BE & TD6_BP & TA_SF & TOA_BE & TOA_BP \\
\hline \multicolumn{8}{|c|}{$\begin{array}{l}\text { D. Prueba de traspaso unitario (estadístico } \\
\text { t) }\end{array}$} \\
\hline Modelo general & $-2,94$ & $-2,83$ & $-4,07$ & $-2,59$ & $-2,69$ & $-3,82$ & $-2,65$ \\
\hline Paridad ajustable & $-3,58$ & $-4,36$ & $-4,72$ & $-4,57$ & $-3,54$ & $-3,91$ & $-3,30$ \\
\hline Banda cambiaria & $-0,72$ & 0,08 & $-1,26$ & 0,58 & 0,45 & $-0,98$ & 0,33 \\
\hline \multicolumn{8}{|c|}{ E. Prueba de igualdad de coeficientes $(B=C)$} \\
\hline Estadístico t & $-2,13$ & $-3,24$ & $-2,59$ & $-3,74$ & $-2,87$ & $-2,16$ & $-2,62$ \\
\hline Probabilidad & 0,03 & 0,00 & 0,01 & 0,00 & 0,00 & 0,03 & 0,01 \\
\hline
\end{tabular}

Fuente: Elaboración propia.

Como lo indica el cuadro 1, el traspaso hacia las tasas promedio de los bancos estatales, tanto pasivas como activas, tiende a ser menor que el efecto a las tasas de los bancos privados. Por otra parte, si se compara el efecto traspaso entre tasas pasivas y activas, se puede concluir que, durante el periodo completo (2000-2013) y el de paridad ajustable (2000-2006), este efecto tendió a ser mayor en las tasas pasivas, aunque durante el periodo de banda cambiaria (2006-2013) parece no haber diferencias significativas. Este último resultado difiere de la investigación de Durán y Esquivel (2008) y Monge y Muñoz (2011), quienes apuntan que el efecto traspaso tiende a ser mayor en las tasas activas en comparación con las pasivas, sin importar el periodo.

El resultado de la aplicación de la prueba de Wald para probar el traspaso unitario (sección D, cuadro 1) señala que únicamente después de la adopción del régimen de banda cambiaria se puede concluir que el efecto traspaso ha sido unitario. Por otra parte, durante el periodo de paridad ajustable, la evidencia estadística apunta a que el traspaso fue menor comparado con el periodo general y con el periodo de banda cambiaria. Para comprobar esto, se aplicó la prueba de Wald, donde la hipótesis nula corresponde a que ambos coeficientes son iguales. De acuerdo con los resultados de las pruebas (sección $\mathrm{E}$, cuadro 1), el efecto traspaso aumentó tras el cambio del régimen cambiario.

Después de la estimación de los efectos de largo plazo, se formulan los modelos de corrección del error, con el fin de estimar las velocidades de ajuste de las tasas de interés ante una perturbación generada por la tasa de política monetaria. Estos modelos se pueden representar con la siguiente forma funcional:

$$
\Delta \mathrm{R}_{\mathrm{t}}^{\mathrm{i}}=\sum_{\mathrm{k}=1}^{\mathrm{n}} \rho_{\mathrm{ik}} \Delta \mathrm{R}_{\mathrm{t}}^{\mathrm{i}}+\sum_{\mathrm{k}=1}^{\mathrm{m}} \alpha_{\mathrm{jk}} \Delta I T P M_{\mathrm{t}}+\delta_{\mathrm{i}} \mu_{\mathrm{t}-1}+\varepsilon_{\mathrm{t}}
$$

Donde $\mu_{\mathrm{t}}$ corresponde al término de error obtenido de las regresiones de largo plazo. De esta forma, los cambios de las tasas de interés dependen de sus valores pasados; $y$ los cambios contemporáneos y pasados, de la tasa de política monetaria.

En el cálculo de la velocidad de ajuste, se empleó la fórmula utilizada por Monge y Muñoz (2011), quienes siguen a Hendry (1995), para calcular el número de periodos promedio en que tardan en realizarse los ajustes, la cual se puede representar como:

$$
\gamma=-\left(\frac{\beta_{2}-\omega_{i}}{\beta_{2} * \delta_{i}}\right)
$$


Donde la velocidad de ajuste $\gamma$ es la diferencia entre el efecto traspaso de largo plazo $\beta_{2}$ y el efecto contemporáneo $\omega_{\mathrm{i}}$, dividido entre el producto del mecanismo de corrección del error $\delta_{\mathrm{i}}(\mathrm{MCE})$ y el efecto traspaso de largo plazo. De esta fórmula, se obtiene la velocidad de ajuste promedio en meses (VA). El resultado de estas estimaciones se presenta en el cuadro 3.

CUADRO 3

COEFICIENTES Y VELOCIDAD DE TRASPASO DEL ITPM A LAS TASAS DEL SISTEMA FINANCIERO PERIODO 2000M1 2013M7

\begin{tabular}{|c|c|c|c|c|c|c|c|}
\hline \multirow[t]{2}{*}{ Periodos } & \multicolumn{4}{|c|}{ Pasivas } & \multicolumn{3}{|c|}{ Activas } \\
\hline & TBP & TP_SF & TD6_BE & TD6_BP & TA_SF & TOA_BE & TOA_BP \\
\hline Efecto contemporáneo & 0,17 & 0,12 & 0,16 & 0,16 & 0,08 & 0,06 & 0,13 \\
\hline Desv. estándar & 0,04 & 0,03 & 0,04 & 0,04 & 0,05 & 0,03 & 0,06 \\
\hline $\mathrm{MCE}$ & $-0,09$ & $-0,09$ & $-0,10$ & $-0,08$ & $-0,08$ & $-0,08$ & $-0,10$ \\
\hline Desv. estándar & 0,02 & 0,02 & 0,02 & 0,02 & 0,02 & 0,02 & 0,02 \\
\hline VA en meses & 8 & 10 & 8 & 10 & 11 & 12 & 8 \\
\hline
\end{tabular}

Fuente: Elaboración propia.

A partir de las estimaciones expuestas en el cuadro 3, es posible concluir que el efecto de un cambio en la tasa de los instrumentos de política monetaria, durante el periodo 2000-2013, tarda entre ocho y doce meses en trasladarse a las otras tasas del sistema financiero. Además, sería razonable pensar que, tras la adopción del nuevo régimen cambiario, estas velocidades hayan cambiado sustancialmente, así como que son distintas dependiendo de si el cambio en la tasa es positivo o negativo. Por estas razones, los próximos apartados de este estudio consideran modelos de corrección del error donde se tomen en cuenta estos factores que pueden influir sobre la velocidad de traspaso.

\section{- $\quad$ Hipótesis de no linealidad}

Con el fin de evaluar si a partir de octubre de 2006 la velocidad de ajuste del efecto traspaso varió por la adopción, de parte del BCCR, de un nuevo arreglo cambiario, se han formulado modelos de corto plazo, con mecanismos de corrección del error, que capten este cambio en la velocidad del ajuste. Esta metodología también fue utilizada por Monge y Muñoz (2011) para probar la misma hipótesis, y ellos concluyeron que la velocidad de ajuste aumentó después de adoptar este régimen. El modelo propuesto tiene la siguiente forma funcional:

$$
\Delta \mathrm{R}_{\mathrm{t}}^{\mathrm{i}}=\sum_{\mathrm{k}=1}^{\mathrm{n}} \rho_{\mathrm{ik}} \Delta \mathrm{R}_{\mathrm{t}}^{\mathrm{i}}+\sum_{\mathrm{k}=0}^{\mathrm{m}} \alpha_{\mathrm{ik}} \Delta I T P M_{\mathrm{t}}+\delta_{\mathrm{i} 1} \mu_{\mathrm{t}-1}+\left(\delta_{\mathrm{i} 1} \mu_{\mathrm{t}-1}\right) \mathrm{d}_{\mathrm{PA}}+\left(\delta_{\mathrm{i} 2} \mu_{\mathrm{t}-1}\right) \mathrm{d}_{\mathrm{BC}}+\varepsilon_{\mathrm{t}}
$$

Donde ahora el término de error de los modelos de largo plazo se separa por medio de variables dicotómicas para poder captar la velocidad en cada periodo. Entonces, si $\delta_{i 2}<\delta_{i 1}$, quiere decir que la velocidad ha disminuido. En el cuadro 4 se observan los resultados de estos modelos. 
CUADRO 4

COEFICIENTES Y VELOCIDAD DE TRASPASO DEL ITPM A LAS TASAS DEL SISTEMA FINANCIERO PERIODO 2000M1 2013M7

\begin{tabular}{|c|c|c|c|c|c|c|c|}
\hline \multirow[t]{2}{*}{ Periodos } & \multicolumn{4}{|c|}{ Pasivas } & \multicolumn{3}{|c|}{ Activas } \\
\hline & TBP & TP_SF & TD6_BE & TD6_BP & TA_SF & TOA_BE & TOA_BP \\
\hline Efecto contemporáneo & 0,18 & 0,13 & 0,16 & 0,16 & 0,09 & 0,07 & 0,13 \\
\hline Desv. estándar & 0,05 & 0,03 & 0,05 & 0,04 & 0,05 & 0,03 & 0,06 \\
\hline \multicolumn{8}{|l|}{ A. Coeficientes } \\
\hline MCE durante paridad ajustable & $-0,07$ & $-0,04$ & $-0,11$ & $-0,07$ & $-0,05$ & $-0,06$ & $-0,07$ \\
\hline Desv. estándar & 0,02 & 0,02 & 0,03 & 0,03 & 0,02 & 0,02 & 0,02 \\
\hline MCE durante banda cambiaria & $-0,11$ & $-0,17$ & $-0,09$ & $-0,08$ & $-0,11$ & $-0,11$ & $-0,12$ \\
\hline Desv. estándar & 0,03 & 0,02 & 0,03 & 0,02 & 0,03 & 0,02 & 0,03 \\
\hline
\end{tabular}

B. Velocidad de ajuste en meses

\begin{tabular}{lccccccc} 
Modelo general & 8 & 10 & 8 & 10 & 11 & 12 & 8 \\
Periodo paridad ajustable & 11 & 20 & 7 & 11 & 16 & 15 & 11 \\
Periodo banda cambiaria & 7 & 5 & 9 & 11 & 9 & 9 & 7 \\
\hline
\end{tabular}

Fuente: Elaboración propia.

De acuerdo con los resultados, la velocidad del ajuste ha aumentado a partir del inicio del sistema de banda cambiaria. Mientras que la velocidad de ajuste en el periodo de paridad ajustable rondaba (dependiendo la tasa) entre los siete y veinte meses; durante el periodo de banda cambiaria, ha oscilado entre cinco y once meses.

Las únicas tasas que no mostraron cambio en la velocidad en meses fueron la tasa de depósitos de los bancos estatales y la de los bancos privados. No obstante, se debe recordar que durante el periodo de banda, el efecto de largo plazo ha resultado ser considerablemente mayor. En esta línea, se podría deducir que se requiere la misma cantidad de meses para trasladar un efecto que estadísticamente es mucho mayor comparado con el periodo de paridad ajustable.

Por consiguiente, se puede observar cómo una mayor flexibilidad cambiaria aumenta el efecto traspaso hacia las tasas de interés del sistema financiero y le brinda al BCCR una mayor capacidad para poder emplear una política monetaria más efectiva.

\section{- $\quad$ Hipótesis de asimetría}

La idea subyacente en esta hipótesis es que, cuando aumenta la tasa de política monetaria, los bancos aumentan las tasas con mayor rapidez; y, por el contrario, cuando la tasa de política cae, los bancos disminuyen las tasas más lentamente.

Para probar esta hipótesis, se crearon dos variables dicotómicas con los siguientes valores:

$$
\text { (6) } d_{(-)}=\left\{\begin{array}{l}
1 \text { si } \mu_{t}<0 \\
0 \text { si } \mu_{t}>0
\end{array} \quad \text { (7) } d_{(+)}=\left\{\begin{array}{l}
1 \text { si } \mu_{t}>0 \\
0 \text { si } \mu_{t}<0
\end{array}\right.\right.
$$


Lo anterior se debe a que, a partir de una situación de igualdad entre ambas partes de esta ecuación, se puede desprender que, ante aumentos del ITPM, se genera una desviación negativa del residuo; $y$, al contrario, si se da una disminución del ITPM, se presenta una desviación positiva del error (Monge y Muñoz, 2011).

$$
\Delta \mathrm{R}_{\mathrm{t}}^{\mathrm{i}}=\sum_{\mathrm{k}=1}^{\mathrm{n}} \rho_{\mathrm{ik}} \Delta \mathrm{R}_{\mathrm{t}}^{\mathrm{i}}+\sum_{\mathrm{k}=0}^{\mathrm{m}} \alpha_{\mathrm{ik}} \Delta \mathrm{ITPM}_{\mathrm{t}}+\left(\delta_{\mathrm{i} 1} \mu_{\mathrm{t}-1}\right)+\left(\delta_{\mathrm{i} 1} \mu_{\mathrm{t}-1}\right) \mathrm{d}_{(-)}+\left(\delta_{\mathrm{i} 2} \mu_{\mathrm{t}-1}\right) \mathrm{d}_{(+)}+\varepsilon_{\mathrm{t}}
$$

Ahora, a partir de los modelos de corrección del error planteados anteriormente, se introducen las nuevas variables creadas. En este caso, si $\delta_{i 1}<\delta_{i 2}$, quiere decir que, ante choques positivos del ITPM (un residuo negativo), el traspaso hacia las tasas de interés se efectúa con mayor rapidez.

Con respecto a esta hipótesis, Durán y Esquivel (2008) no hallaron evidencia de asimetría en el traspaso para Costa Rica. En la investigación de Monge y Muñoz (2011), los coeficientes $\delta_{i 1}$ y $\delta_{\mathrm{i} 2}$ no resultaron estadísticamente distintos, pero lograron establecer algunas conclusiones a partir de la cantidad de meses promedio que tardan en ajustarse las tasas ante choques de la tasa de política monetaria.

CUADRO 5

ASIMETRÍA EN LA VELOCIDAD DEL TRASPASO DEL ITPM A LAS TASAS DEL SISTEMA FINANCIERO MODELOS DE CORTO PLAZO PERIODO 2000M1 2013M7

\begin{tabular}{|c|c|c|c|c|c|c|c|}
\hline \multirow[t]{2}{*}{ Periodos } & \multicolumn{4}{|c|}{ Pasivas } & \multicolumn{3}{|c|}{ Activas } \\
\hline & TBP & TP_SF & TD6_BE & TD6_BP & TA_SF & TOA_BE & TOA_BP \\
\hline $\begin{array}{l}\text { Coeficiente de traspaso de corto } \\
\text { plazo }\end{array}$ & 0,16 & 0,12 & 0,16 & 0,15 & 0,07 & 0,06 & 0,11 \\
\hline Desv. estándar & 0,04 & 0,03 & 0,04 & 0,03 & 0,04 & 0,03 & 0,05 \\
\hline
\end{tabular}

A. Modelo con asimetría

$\begin{array}{lccccccc}\text { Desviaciones negativas } \delta_{\mathrm{i} 1} & -0,14 & -0,08 & -0,11 & -0,16 & -0,13 & -0,09 & -0,17 \\ \text { Desv. estándar } & 0,03 & 0,03 & 0,03 & 0,03 & 0,03 & 0,03 & 0,03 \\ \text { Desviaciones positivas } \delta_{\mathrm{i} 2} & -0,08 & -0,09 & -0,09 & -0,04 & -0,05 & -0,07 & -0,07 \\ \text { Desv. estándar } & 0,02 & 0,02 & 0,02 & 0,02 & 0,02 & 0,02 & 0,02\end{array}$

B. Velocidad de ajuste en meses

\begin{tabular}{lccccccc} 
Modelo general & 8 & 10 & 8 & 10 & 11 & 12 & 8 \\
Ante choques negativos & 6 & 11 & 7 & 5 & 7 & 10 & 5 \\
Ante choques positivos & 10 & 9 & 9 & 19 & 17 & 12 & 12 \\
\hline
\end{tabular}

Fuente: Elaboración propia.

En el caso del presente estudio, no se pudo comprobar que ambos coeficientes son distintos para el caso de las tasas pasivas y activas de los bancos estatales, así como para la tasa pasiva promedio del sistema financiero (ver anexo 4) en tanto que, para la tasa activa promedio del sistema financiero (TA_SF) y la tasa activa para otras actividades de los bancos privados (TOA_BP), la diferencia promedio es hasta de ocho meses (cuadro 5). 
En la tasa activa promedio del sistema financiero, ante choques negativos (un aumento del ITPM), la velocidad promedio es de siete meses $y$, ante choques positivos (una disminución del ITPM), el traspaso tarda diecisiete meses en promedio. Si se compara con la velocidad de la tasa pasiva promedio del sistema financiero, de nueve a once meses, se puede concluir que los bancos aumentan con mayor rapidez las tasas activas que las tasas pasivas, con el posible propósito de aumentar su margen de intermediación financiera por un determinado periodo de tiempo.

Esto sucede de igual forma para el caso de las tasas activas de los bancos privados, donde, ante un aumento del ITPM, se tarda en promedio cinco meses en trasladar su efecto; mientras que, ante disminuciones, se tarda doce meses en promedio.

Con respecto a las tasas pasivas, no se halló evidencia de que, ante disminuciones del ITPM, los bancos reaccionaron con mayor rapidez. Para el caso de la TP_SF y la TD6_BE, no se puede concluir que ambos coeficientes sean estadísticamente distintos; $y$ para el caso de la TBP y la TD6_BP, se encontró que reaccionan con mayor rapidez ante aumentos del ITPM. Sin embargo, no es posible afirmar que las tasas activas reaccionan con mayor rapidez antes choques negativos o positivos (con excepción de los bancos estatales).

Asimismo, se estimaron modelos donde se prueba la hipótesis de asimetría y no linealidad simultáneamente. Los resultados son bastante similares a los anteriores y no modifican de forma sustancial ninguna de las conclusiones presentadas, aunque se aprecia una disminución en la velocidad de meses promedio que tarda en ajustarse el efecto traspaso al adoptarse el nuevo régimen cambiario, tanto en choques positivos como negativos. El cuadro con los resultados de las estimaciones se incluye en el anexo 5.

\section{- $\quad$ Factores que interrumpen el efecto traspaso}

En esta sección se analizarán los factores que pueden interferir en el proceso de transmisión de los cambios en las tasas de interés y que, por lo tanto, afectan la efectividad de la política monetaria. Primero, se comentará la definición de las variables empleadas en el análisis y, posteriormente, se expondrán los resultados obtenidos.

\section{- $\quad$ Descripción de las variables}

Para disponer de una medida cuantitativa que capte la evolución de las variables de dolarización y concentración bancaria, se emplearon varias metodologías. En el caso de la dolarización del sistema financiero, se recurrió al nivel de depósitos en dólares como porcentaje del total de depósitos de todo el sistema financiero. Por su parte, para obtener una medida de concentración bancaria, se usó el índice de Herfindahl-Hirschman de concentración de los activos (a partir de ahora índice de concentración bancaria) del sistema financiero, en el cual se utilizaron las principales veinte entidades financieras ${ }^{8}$.

Los datos del saldo primario del Gobierno Central se encuentran en formato mensual desde enero de 2006. Con el fin de obtener el resultado primario del Gobierno Central y el del Banco Central de Costa Rica como porcentaje el producto interno bruto nominal (PIB), se utiliza la serie mensual de producto interno bruto nominal calculada por el BCCR.

8 Las entidades utilizadas en dicho cálculo fueron: Banco General, Bansol, Banco Improsa, Citibank, Bac San José, Banco Lafise, Mucap, Mutual Alajuela, Coocique, Cooperservidores, Coopealizanza, Banco Nacional, Banco de Costa Rica, Banco Crédito Agrícola de Cartago, Banco Popular, Banco Davivienda, Scotiabank, Banco Promerica, Banco Cathay, Banco BCT. 


\begin{tabular}{ll}
\hline Abreviatura & Variable \\
\hline DOL & Nivel de dolarización de los depósitos del sistema financiero \\
HH_ACT & Índice de concentración de activos del sistema financiero \\
DF & Resultado primario del Gobierno Central como porcentaje (\%) del PIB \\
DF_BCCR & Resultado financiero del Banco Central de Costa Rica como \% del PIB \\
\hline
\end{tabular}

Después de definir cada variable, se evaluó cada efecto por separado. Para esto, se utilizaron modelos donde se incluyeron las interacciones entre la tasa de política monetaria con cada uno de los distintos efectos descritos anteriormente. Si la interacción resulta significativa y negativa, quiere decir que el efecto traspaso se ve reducido.

El signo esperado de la dolarización, de la concentración bancaria, del saldo primario del Gobierno Central y del resultado del BCCR es negativo, ya que todos representan barreras para la transmisión de los cambios en la tasa de los instrumentos de política monetaria del BCCR. Así, aumentos en estas variables afectarían negativamente el grado de traspaso de la tasa de política monetaria hacia las tasas de interés del sistema financiero.

\section{- Dolarización}

La literatura económica reconoce que altos niveles de dolarización disminuyen la efectividad de la política monetaria. Siguiendo esta línea, Rossini y Vega (2007) sugieren que disminuir los niveles de dolarización financiera puede reforzar el efecto traspaso de la tasa de interés de política monetaria.

Para estimar este efecto de la dolarización, se siguió lo propuesto por Medina et al. (2011), quienes estiman los efectos de la dolarización para los países de Centroamérica y los denominados LA6, por medio de las interacciones de la tasa de política y el nivel de dolarización financiera.

$$
R_{i t}=\beta_{i 1}+\beta_{i 2} I T P M_{t}+\beta_{i 3} I T P M_{t} D O L_{t}+\varepsilon_{i t}
$$

Donde $D O L_{t}$ se refiere al nivel de dolarización financiera y el coeficiente $\beta_{i 3}$ representa el efecto del nivel de dolarización sobre el coeficiente de traspaso del ITPM, que debe ser menor o igual a cero. Es de esperar que, cuando el nivel de dolarización financiera tiende a cero, el efecto traspaso de largo plazo tenderá al coeficiente $\beta_{i 2}$. El cuadro 6 contiene las estimaciones del coeficiente $\beta_{i 3}$.

$$
\begin{gathered}
\text { CUADRO } 6 \\
\text { INTERACCIÓN ENTRE LA DOLARIZACIÓN Y EL ITPM (MULTIPLICADO POR 100) } \\
\text { ENERO DE 2000-JULIO DE } 2013
\end{gathered}
$$

\begin{tabular}{lccc}
\hline Variable & Coeficiente & Desviación estándar & Estadístico t \\
\hline TBP & $-1,08$ & 0,22 & $-4,83$ \\
TP_SF & $-1,02$ & 0,20 & $-4,98$ \\
TD6_BE & $-1,46$ & 0,21 & $-7,07$ \\
TD6_BP & $-1,39$ & 0,12 & $-11,28$ \\
Promedio & $-1,24$ & & $-4,58$ \\
TA_SF & $-0,84$ & 0,18 & $-5,77$ \\
TOA_BE & $-1,20$ & 0,21 & $-2,92$ \\
TOA_BP & $-0,75$ & 0,26 & \\
Promedio & $-0,93$ & & \\
\hline
\end{tabular}

Fuente: Elaboración propia. 
Como lo indica el cuadro 6 , en general el coeficiente $\beta_{i 3}$ ronda alrededor de la unidad y el efecto tiende a ser menor en las tasas activas. De forma específica, un aumento (disminución) de un punto porcentual (p.p.) en la dolarización financiera causa una disminución (aumento) de 1,46\% del efecto traspaso del ITPM sobre la tasa promedio de depósitos a seis meses plazo de los bancos estatales, $y$ de un 1,39\% de los bancos privados. Por otra parte, este efecto es de 1,08\% para la tasa básica pasiva y de 1,02\% para la tasa pasiva promedio del sistema financiero.

Por otro lado, en el caso de las tasas activas, un aumento (disminución) de un punto porcentual en la dolarización causa una disminución (aumento) del efecto traspaso del ITPM de 1,20\% sobre la tasa activa para otras actividades de los bancos estatales. Este efecto disminuye considerablemente en el caso de la tasa promedio para otras actividades de los bancos privados, cuyo efecto es de $0,75 \%$.

\section{- $\quad$ Concentración bancaria}

Al igual que la dolarización, la concentración bancaria puede influir en la efectividad de la política monetaria. Por ejemplo, es de esperar que mercados poco competitivos reaccionen en menor medida ante cambios en la tasa de política monetaria. En Costa Rica, la concentración bancaria ha disminuido de manera significativa en los últimos veinte años (ver anexo 6). Sin embargo, el nivel de concentración sigue siendo lo suficientemente alto como para pensar que no se trata de un mercado altamente competitivo $y$, por ende, que podría tener efectos sobre la efectividad de transmisión de los cambios en el instrumento de política del BCCR.

Para medir el impacto de la concentración bancaria sobre el instrumento de política del BCCR, se formuló un modelo que capta la interacción entre la tasa de política monetaria y el logaritmo del índice de concentración bancaria. El modelo utilizado tiene la siguiente forma funcional:

$$
R_{i t}=\beta_{i 1}+\beta_{i 2} I T P M_{t}+\beta_{i 3} I T P M_{t} \log \left(H H_{-} A C T_{t}\right)+\varepsilon_{i t}
$$

Donde las tasas de interés del sistema financiero ahora también estarán en función de la interacción entre la tasa de política monetaria y el logaritmo del índice de concentración bancaria. El coeficiente $\beta_{i 3}$ representa el efecto del nivel de concentración bancaria sobre el traspaso de largo plazo. De esta forma, el efecto traspaso está en función del nivel de concentración bancaria:

$$
\frac{\partial R_{i t}}{\partial I T P M_{t}}=\beta_{i}^{*}=\beta_{i 2}+\beta_{i 3} \log \left(H H_{-} A C T_{t}\right)
$$

El coeficiente $\beta_{i 3}$ deber ser menor o igual a cero. De esta manera, con un aumento (disminución) de un $1 \%$ en el nivel de concentración bancaria, el efecto traspaso final ( $\left.\beta^{*}\right)$ disminuirá (aumentará) en $\beta_{i 3}$ puntos porcentuales. El cuadro 7 muestra las estimaciones del coeficiente $\beta_{3}$.

CUADRO 7

INTERACCIÓN DE LA TASA DE POLÍTICA MONETARIA (ITPM) Y EL LOGARITMO DEL ÍNDICE DE CONCENTRACIÓN BANCARIA ENERO DE 2000-JULIO DE 2013

\begin{tabular}{lcc}
\hline Var. dependiente & Coeficiente $\beta_{i 3}$ & Desviación estándar \\
\hline TBP & $-0,15$ & 0,036 \\
TP_SF & $-0,13$ & 0,041 \\
TD6_BE & $-0,13$ & 0,034 \\
TD6_BP & $-0,16$ & 0,030 \\
\hline
\end{tabular}


Continuación...

\begin{tabular}{lcc}
\hline Var. dependiente & Coeficiente $\beta_{i 3}$ & Desviación estándar \\
\hline Promedio & $-0,14$ & 0,053 \\
TA_SF & $-0,22$ & 0,082 \\
TOA_BE & $-0,22$ & 0,077 \\
TOA_BP & $-0,24$ & \\
Promedio & $-0,23$ & \\
\hline
\end{tabular}

Fuente: Elaboración propia.

A partir del cuadro anterior, se obtiene el resultado de que un cambio de un $1 \%$ en el nivel de concentración bancaria genera un cambio promedio de $0,14 \%$ en el efecto traspaso de la tasa de interés de la política monetaria hacia las tasas de interés pasivas. También, se observa que este efecto tiende a ser mayor en las tasas activas, donde un cambio porcentual de un $1 \%$ en el nivel de concentración bancaria ocasiona un cambio promedio de $0,23 \%$ hacia las tasas activas. Finalmente, no se encontraron diferencias significativas entre las tasas de interés — pasivas y activas- de los bancos privados y las de los bancos estatales.

\section{- $\quad$ Resultado primario del Gobierno Central}

En el momento de diseñar la política monetaria, las autoridades deben tomar en cuenta la situación fiscal del Gobierno Central, por cuanto las acciones que tomen las autoridades fiscales podrían ser contradictorias con respecto al objetivo de la autoridad monetaria. Políticas económicas que van en direcciones opuestas pueden terminar resultando inefectivas e, incluso, perjudiciales para la economía. En este caso, el saldo primario como proporción del PIB puede interferir en el efecto traspaso cuando esfuerzos de las autoridades monetarias por influir en las tasas de interés se ven afectados por la necesidad de financiamiento interno de las autoridades fiscales. Adicionalmente, altos niveles de déficit fiscal pueden aumentar la prima de riesgo y, por último, también aumentar las tasas de interés. Todo esto puede interferir en el efecto traspaso.

Por ejemplo, si el Banco Central disminuye la tasa de política monetaria con el fin de bajar las tasas de interés en el sistema financiero y así influir positivamente sobre la demanda agregada, esto puede resultar menos efectivo si, al mismo tiempo, el Gobierno Central capta recursos internos $y$, con ello, eleva las tasas de interés.

Para estimar este efecto, se plantea un modelo similar a los anteriores, donde se incluyen las interacciones de la tasa de política monetaria con el déficit fiscal interanual en el momento $t$.

$$
R_{i t}=\delta_{i 1}+\delta_{i 2} I T P M_{t}+\delta_{i 3} D F_{t}+\delta_{i 4} I T P M_{t} D F_{t}+\delta_{i 5} R_{i(t-1)}+\varepsilon_{i t}
$$

Donde cada tasa de interés del sistema financiero se encuentra en función de la tasa de política monetaria, la relación resultado primario-PIB, la interacción entre el ITPM y el resultado primario y su valor pasado. El cuadro 8 resume la estimación de estos modelos.

El periodo analizado fue de enero de 2007 a julio de 2013 (con un total de 79 observaciones). La razón fundamental por la cual se decidió utilizar este periodo se debe a que los datos mensuales del resultado fiscal están disponibles únicamente a partir del año $2006^{9}$.

\footnotetext{
9 La limitada disponibilidad de datos se debe a un cambio metodológico que dificulta su comparación, antes de esta fecha las estadísticas corresponden a un flujo de caja mientras que posteriormente corresponden a cifras devengadas.
} 
Como resultado de que las tasas de interés de mercado también están en función del resultado fiscal primario, el efecto traspaso de largo plazo está en función también de la situación fiscal en el periodo $t$. Esto se puede escribir como la primera derivada parcial de la ecuación 12 con respecto a la tasa de política:

$$
\frac{\partial R_{i t}}{\partial I T P M_{t}}=\delta_{i 1}^{*}=\delta_{i 2}+\delta_{i 4} D F_{t}
$$

Donde los coeficientes $\delta_{i 4}$ corresponden al efecto que ejerce el resultado fiscal sobre el efecto traspaso de largo plazo. Como resultado general, se puede concluir, a partir del cuadro 8, que en efecto la situación fiscal afecta de forma negativa la magnitud final del coeficiente de traspaso del ITPM. Este efecto es mayor en las tasas activas comparadas con las tasas pasivas y podría señalar que el efecto estrujamiento del crédito es más fuerte para los demandantes de recursos que para los oferentes.

\section{CUADRO 8}

INTERACCIONES ENTRE EL SALDO PRIMARIO DEL GOBIERNO CENTRAL Y EL ITPM ENERO DE 2007-JULIO DE 2013

\begin{tabular}{lccc}
\hline Tasa de interés & Coeficiente & Desviación estándar & Estadístico t \\
\hline TBP & $-0,031$ & 0,013 & $-2,45$ \\
TP_SF & $-0,023$ & 0,008 & $-2,86$ \\
TD6_BE & $-0,031$ & 0,011 & $-2,80$ \\
TD6_BP & $-0,018$ & 0,009 & $-2,09$ \\
& & & $-2,23$ \\
Promedio pasivas & $-0,026$ & & $-2,48$ \\
& & 0,013 & $-2,29$ \\
TA_SF & $-0,029$ & 0,014 & \\
TOA_BE & $-0,035$ & 0,018 & \\
TOA_BP & $-0,042$ & & \\
Promedio activas & & & \\
\hline
\end{tabular}

Fuente: Elaboración propia.

Se puede observar que la tasa con un menor coeficiente de interacción (en términos absolutos) es la tasa de depósitos a seis meses plazo de los bancos privados, donde un aumento de 1 p.p. en el resultado fiscal (déficit) genera una disminución de 1,8\% en el efecto traspaso de largo plazo. Por otra parte, la tasa con el mayor coeficiente de interacción corresponde a la tasa para otras actividades de los bancos privados, donde un aumento de 1 p.p. en el resultado fiscal (déficit) genera una disminución de 4,2\% en el efecto traspaso de largo plazo. Por último, no existe una tendencia clara en cuanto si el resultado fiscal afecta en mayor o menor medida el efecto traspaso hacia los bancos privados o hacia los bancos estatales, pero sí una tendencia a tener un mayor impacto en las tasas de interés activas.

\section{- Déficit del BCCR}

El resultado financiero negativo del banco central también puede disminuir la efectividad del mecanismo de transmisión. Laurens (2005) explica este efecto diciendo que, por ejemplo, los prés- 
tamos directos del Banco Central al Gobierno afectan el resultado financiero del Banco Central y limitan la capacidad del banco para señalar de forma clara la orientación de la política monetaria, al elevar las tasas de interés y realizar operaciones de mercado abierto. Este es el mismo efecto que produce un tipo de cambio fijo que obliga a un Banco Central a realizar operaciones de esterilización. Por estas razones, el déficit del BCCR se puede utilizar como un indicador del grado de independencia para llevar a cabo la política monetaria que tiene el Banco.

Para realizar las estimaciones, se formularon modelos similares a los anteriores, donde cada tasa de interés del sistema financiero está en función de la tasa de política monetaria y la interacción con el déficit del $\mathrm{BCCR}^{10}$. Como consecuencia de lo anterior, el efecto traspaso de largo plazo dependerá de la interacción del ITPM con el déficit del Banco Central.

$$
R_{i t}=\gamma_{i 1}+\gamma_{i 2} I T P M_{t}+\sum_{K=1}^{2} \gamma_{i 3 k} I T P M_{t} D F \_B C C R_{t} D_{t k}+\gamma_{i 4} R_{i(t-1)}+\varepsilon_{i t}
$$

Donde $D_{t k}$ representa la variable dicotómica $k$ en el momento $t, D F \_B C C R_{t}$ el déficit del Banco Central de Costa Rica como porcentaje del PIB y $\gamma_{i 3 k}$ el coeficiente o efecto que ejerce el resultado sobre el efecto traspaso de largo plazo hacia la tasa $i$. El resumen de estos coeficientes se presenta en el cuadro 9.

CUADRO 9

INTERACCIÓN ENTRE EL DÉFICIT DEL BANCO CENTRAL Y EL ITPM

ENERO DE 2000-JULIO DE 2013

\begin{tabular}{lccc}
\hline Tasa de interés & General & Def $\leq 1 \%$ & Def $>1 \%$ \\
\hline TBP & & & 0,02 \\
Desv. estándar & 0,02 & 0,04 & $-0,06$ \\
TP_SF & $-0,06$ & $-0,08$ & 0,01 \\
Desv. estándar & 0,01 & 0,03 & $-0,08$ \\
TD6_BE & $-0,08$ & $-0,11$ & 0,02 \\
Desv. estándar & 0,02 & 0,04 & $-0,10$ \\
TD6_BP & $-0,10$ & $-0,12$ & 0,02 \\
Desv. estándar & 0,02 & 0,03 & $-0,09$ \\
& & & $-0,07$ \\
Promedio pasivas & $-0,09$ & $-0,11$ & 0,02 \\
& & & $-0,09$ \\
TA_SF & $-0,07$ & $-0,08$ & 0,03 \\
Desv. estándar & 0,02 & 0,03 & $-0,07$ \\
TOA_BE & $-0,09$ & $-0,08$ & 0,02 \\
Desv. estándar & 0,03 & 0,04 & $-0,08$ \\
TOA_BP & $-0,04$ & $-0,14$ & 0,04 \\
Desv. estándar & 0,02 & $-0,10$ & \\
Promedio activas & $-0,07$ & 0,00 & \\
\hline
\end{tabular}

Fuente: Elaboración propia.

10 Como porcentaje del producto interno bruto nominal. 
Con base en el cuadro 9, se puede concluir que la situación financiera del Banco Central de Costa Rica afecta de forma significativa el efecto traspaso del ITPM hacia la tasas de interés de mercado.

Se observa que un aumento o disminución de diez puntos base en el déficit del Banco Central de Costa Rica genera una disminución o aumento (respectivamente) de 1,1\% del traspaso hacia la tasa básica pasiva. Utilizando la prueba de igualdad de coeficientes, se halló una pequeña diferencia, pero significativa, entre el efecto sobre la tasa activa y sobre la tasa pasiva promedio del sistema financiero; esta última es la que menos reacciona ante cambios en el resultado financiero del Banco Central como proporción del PIB (ver anexo 7).

Por otra parte, en las tasas de depósitos y las activas para otras actividades se encontraron diferencias significativas entre los bancos estatales y privados. Se determinó que los bancos privados en promedio tienen un coeficiente de interacción menor, por lo cual cambios en los resultados financieros del BCCR tienden a afectar menos el efecto traspaso hacia las tasas activas de los bancos privados, con respecto a la tasas pasivas. Ante un aumento de diez puntos base del déficit del BCCR, el efecto traspaso hacia las tasas de depósitos de los bancos privados se disminuye en $1 \%$. El efecto es mucho menor en la tasa de otras actividades de los bancos privados, donde, con un aumento de diez puntos base del déficit del BCCR, el efecto traspaso disminuye 0,40\%.

Por último, no se encontró evidencia estadística para concluir que la distorsión del déficit del BCCR sobre el efecto traspaso de largo plazo sea mayor o menor dependiendo de la magnitud del déficit (ver anexo 8).

\section{- $\quad$ Estimación conjunta para todos los factores}

Por último, con el fin de disponer de medidas cuantitativas que ayuden a discriminar la relación de cada factor individual sobre el efecto traspaso controlando por los efectos de los demás factores, se procedió a realizar un grupo de estimaciones con todos los factores para todo el periodo de estudio. Las estimaciones se realizaron para las principales tasas de interés, esto es, para la tasa básica pasiva y las tasas activa y pasiva promedio del sistema financiero.

Con estas estimaciones se espera observar el efecto de cada una de los factores sobre el coeficiente del traspaso de la tasa de política sin ignorar el posible efecto de los otros factores. Como consecuencia de lo anterior, el efecto traspaso de largo plazo dependerá de la interacción del ITPM con cada uno de los factores especificados anteriormente en esta sección, tal y como lo especifica la ecuación 15.

$$
\begin{gathered}
R_{i t}=\beta_{i 1}+\beta_{i 2} I T P M_{t}+\beta_{i 3} I T P M_{t} D O L_{t}+\beta_{i 4} I T P M_{t} \log \left(H H_{-} A C T_{t}\right)+\beta_{i 5} I T P M_{t} D F_{t}+ \\
\beta_{i 6} I T P M_{t} D F_{-} B C C R_{t} D_{t k}+\varepsilon_{i t}
\end{gathered}
$$

Los resultados que se muestran en el cuadro 10 señalan que coherente con las estimaciones anteriores, cada uno de los factores reduce el efecto traspaso de la tasa de política monetaria; sin embargo, el factor que reduce con mayor fortaleza el traspaso es el déficit del Banco Central, seguido del saldo del Gobierno Central. Cabe señalar que las estimaciones individuales también mostraron que estos son los dos principales factores en términos de reducción del efecto traspaso, aunque en ese caso el saldo del Gobierno Central tenía el principal efecto seguido de cerca por el déficit del BCCR. 


\section{CUADRO 10 \\ EFECTO DE CADA INTERACCIÓN EN ESTIMACIÓN CONJUNTA ENERO DE 2007-JULIO DE 2013}

\begin{tabular}{lccc}
\hline Factor & TBP & TP_SF & TA_SF \\
\hline Efecto Traspaso TPM & $0,819^{*}$ & $1,076^{* * *}$ & $1,286^{* * * *}$ \\
Índice de Concentración & $-0,006^{* * *}$ & $-0,004^{* * * *}$ & $-0,001^{* * *}$ \\
Saldo Primario Gob, Central & $-0,050^{* * *}$ & $-0,025^{* * *}$ & $-0,053^{* * *}$ \\
Dolarización & $-0,001$ & $-0,005^{* *}$ & $-0,011^{* * *}$ \\
Déficit del BCCR & $-0,349^{* * *}$ & $-0,381^{* * *}$ & $-0,345^{* * * *}$ \\
\hline
\end{tabular}

Fuente: Elaboración propia , *, **, *** significativo al 10\%, 5\%, 1\% respectivamente.

\section{CONCLUSIONES}

El propósito de esta investigación es explorar la efectividad del instrumento de política monetaria del BCCR para transmitir sus movimientos al resto de las tasas de interés del sistema financiero, así como estudiar los distintos factores que limitan este traspaso. Este es el primer estudio del tema desde que el BCCR hizo oficial la tasa de política monetaria como una tasa de referencia en el mercado integrado de liquidez (MIL). También fue posible evaluar este efecto junto con la adopción de un arreglo cambiario de banda, y medir cómo un mayor grado de libertad del BCCR para manejar su política monetaria puede influir en el traspaso de cambios en la TPM las tasas de interés del sistema financiero.

Los modelos de largo plazo para el efecto traspaso bajo la metodología de Engle-Granger permitieron encontrar que este efecto experimentó una variación positiva a partir del año 2006, fecha de la adopción del nuevo régimen cambiario. Además, se concluyó que, tras la adopción de la banda cambiaria, el efecto traspaso ha sido unitario.

Utilizando modelos de corrección de error, se calculó que en promedio, durante el periodo 2000-2013, el efecto traspaso de largo plazo tarda entre ocho y doce meses en trasladarse a las tasas del sistema financiero. Adicionalmente, se formularon modelos que captaron las velocidades antes y después del cambio en el régimen cambiario, que indican que la velocidad de ajuste ha aumentado a partir de la implementación del régimen de bandas cambiarias. La velocidad de ajuste en el periodo de paridad ajustable rondaba entre siete $y$ veinte meses, $y$ durante el periodo de banda cambiaria ha oscilado entre cinco y once meses, según la tasa. Del análisis de la hipótesis de asimetría se puede concluir que el sistema financiero aumenta con mayor rapidez las tasas activas que las tasas pasivas, muy probablemente con el fin de aumentar su margen de intermediación financiera.

Este estudio también examinó los distintos factores que pueden afectar el efecto traspaso de la tasa de política hacia las tasas del sistema financiero. Las estimaciones indican que un aumento de $1 \%$ en el nivel de dolarización reduce en $1,46 \%$, el efecto traspaso. Un incremento en la concentración bancaria de un $1 \%$ afecta negativamente en $0,14 \%$ el efecto traspaso. Así mismo un deterioro de $1 \%$ el resultado del Gobierno Central o del resultado financiero del BCCR, ambos como proporción del PIB, reducen en 1,8\% y 1,1\%, respectivamente, el efecto traspaso. En resumen, todos los factores poseen una relación negativa con el efecto traspaso y si bien son resultados esperados teóricamente, nunca antes habían sido medidos en estudios de este tipo.

Cabe señalar que a pesar de que en periodos previos al año 2011 el BCCR no tenía un instrumento como el Mercado Integrado de Liquidez (MIL), para emplear de forma efectiva la tasa de política monetaria; esto no implica que en periodos previos el ente emisor haya dejado de hacer política monetaria, sino que la hacía con los instrumentos disponibles en el momento (tal y como lo reseña 
la ITPM) y que, aunque podrían estar limitados por el régimen cambiario (Trinidad Imposible), no implicaban su inexistencia y esto es también un hallazgo importante de este estudio.

Estos resultados evidencian que se podría mejorar la efectividad de la tasa de política monetaria como instrumento si algunas distorsiones de la economía se reducen o se eliminan, principalmente el déficit del BCCR. Por tanto, es evidente que las condiciones macroeconómicas son determinantes para la efectividad de la política. Por último, los autores reconocen el carácter exploratorio de este estudio $y$ la necesidad de continuar explorando este tema para analizar temas como el orden del traspaso, el efecto de la alta dolarización en los créditos, así como los periodos de alta intervención en el mercado cambiario.

\section{REFERENCIAS}

Alvarez-Plata , P., y A. García-Herrero. (2007). To dollarize or de-dollarize:Consequences for Monetary Policy. Paper prepared for the Asian Development Bank.

Borio, C., y W. Fritz. (1995). The Response of Short Term Bank Lending Rates to Policy Rates: A Cross-Country Perspective. Monetary and economic Departament. Bank for International Settlements.

Castro, A., y C. Chaverri. (2013). Indicador de tasa de política monetaria del Banco Central de Costa Rica. San José: DT-11-2013. Departamento de Investigación Económica. Banco Central de Costa Rica.

Cottarelli , C., y A. Kourelis. (1994). Financial Structure, Bank Lending Rates, and the Transmission Mechanism of Monetary Policy. IMF Staff Papers Vol.41. No.4, International Monetary Fund.

Durán-Víquez, R., y M. Esquivel-Monge. (2008). Policy Rate Pass-Through: Evidence From The Costa rican Economy. Documento de Investigación: DIE-05-2008-DI. Departamento de Investigación, Banco Central de Costa Rica.

Engle, R., y C. Granger. (1987). Co-Integration and Error Correction: Representation, Estimation, and Testing. Econometrica, Vol. 55, No. 2. , 251-276.

Espinoza Vega, M., y A. Rebucci. (2003). Retail Bank Interest Rate Pass-Through: Is Chile Atypical? International Monetary Fund (IMF).

Laurens, B. (2005). Monetary Policy Implementation at Different Stages of Market Development. Washington: International Monetary Fund: IMF Occasional Paper No. 244.

Mangwengwende, T., Chinzara, Z., y H. Nel. (2011). Bank concentration and the interest rate passthrough in Sub-Saharan African countries. Working paper 233. Department of Economics, Rhodes University.

Medina Cas, S., Carrión-Menéndez, A., y F. Frantischek. (2011). The Policy Interest-Rate PassThrough in Central America. International Monetary Fund (IMF Working Paper:Western Hemisphere Department).

Monge Badilla, C., y E. Muñoz Salas. (2011). El traspaso de cambios en la tasa de interés de política monetaria hacia las tasas de interés delsistema financiero costarricense. Documento de Investigación DEC-DIE-DI-03-2011. Departamento de Investigación, Banco Central de Costa Rica.

Roelands, S. (2013). Asymmetric Interest Rate Pass-Through from Monetary Policy: The Role of Bank Regulation. Notre Dame, Inglaterra: Department of Economics, University of Notre Dame.

Rossini, R., M. Vega. (2007). The monetary policy transmission mechanism under financial dollarisation: the case of Peru 1996-2006. Central Bank of Peru. 


\section{Anexo 1}

La variable ITPM se construyó a partir de la investigación de Castro y Chaverri (2013), quienes construyen un indicador de tasa de política monetaria que lo constituye:

- Enero de 2000-febrero de 2006: Tasa del sistema de inversiones de corto plazo (SICP) a siete días plazo. Se consideró utilizar dicha tasa porque, en un inicio, no se contaba con el instrumento a un día plazo. No obstante, cuando se creó la opción de un día, la tasa se definió igual a la tasa de siete días.

- Marzo de 2006-agosto 2007: Tasa del SICP a un día plazo. A partir de marzo del 2006, existe de forma oficial una tasa de un día plazo.

- Setiembre de 2007-octubre de 2010: Tasa un día plazo (DON) de Central Directo, momento en que deja de funcionar el SICP. Central Directo se constituye como una plataforma electrónica que le permite al BCCR captar recursos del público a diferentes plazos.

- Noviembre de 2010-mayo de 2011: Tasa promedio de la sesión en el MIL. A partir de esta fecha, existe tanto la facilidad permanente de depósito como la facilidad permanente de crédito.

- Junio de 2011-julio de 2013: Definición actual de tasa de política monetaria.

\section{Anexo 2}

\section{CUADRO 1}

PRUEBA DE CAUSALIDAD EN EL SENTIDO DE GRANGER PROBABILIDAD DE NO RECHAZAR HO PERIODO ENERO DE 2000-JULIO DE 2013

\begin{tabular}{lcc}
\hline \multirow{2}{*}{ Tasa $\mathrm{j}$} & \multicolumn{2}{c}{ Hipótesis nula } \\
\cline { 2 - 3 } & Tasa j no causa ITPM & ITPM no causa tasa j \\
\hline TP_SF & 0,00 & 0,09 \\
TBP & 0,00 & 0,16 \\
TD6_BE & 0,00 & 0,13 \\
TD6_BP & 0,00 & 0,00 \\
TA_SF & 0,02 & 0,00 \\
TOA_BE & 0,01 & 0,07 \\
TOA_BP & 0,03 & 0,00 \\
\hline
\end{tabular}

Fuente: Elaboración propia. 
CUADRO 2

PRUEBA DE CAUSALIDAD EN EL SENTIDO DE GRANGER PROBABILIDAD DE NO RECHAZAR HO PERIODO ENERO DE 2000-JULIO DE 2013

\begin{tabular}{cc}
\hline & Hipótesis nula \\
\hline TD6_BE no causa TOA_BE & TOA_BE no causa TD6_BE \\
0,00 & 0,12 \\
TD6_BP no causa TOA_BP & TOA_BP no causa TD6_BP \\
0,00 & 0,89 \\
TP_SF no causa TA_SF & TA_SF no causa TP_SF \\
0,00 & 0,52 \\
\hline
\end{tabular}

Fuente: Elaboración propia.

\section{CUADRO 3}

PRUEBA DE CAUSALIDAD EN EL SENTIDO DE GRANGER PROBABILIDAD DE NO RECHAZAR HO PERIODO ENERO DE 2000-JULIO DE 2013

\begin{tabular}{cc}
\hline \multicolumn{2}{c}{ Hipótesis nula } \\
\hline TD6_BE no causa TD6_BP & TD6_BP no causa TD6_BE \\
0,00 & 0,00 \\
TOA_BE no causa TOA_BP & TOA_BP no causa TOA_BE \\
0,00 & 0,27 \\
\hline
\end{tabular}

Fuente: Elaboración propia.

\section{Anexo 3}

CUADRO 1

GRADO DE INTEGRACIÓN DE LAS VARIABLES HIPÓTESIS NULA: PRESENCIA DE RAÍZ UNITARIA* PERIODO ENERO DE 2000-JULIO DE 2013

\begin{tabular}{|c|c|c|c|c|c|c|c|}
\hline \multirow{2}{*}{ Tasas } & \multicolumn{3}{|c|}{ Variables en nivel } & \multicolumn{3}{|c|}{ Variables en primera diferencia } & \multirow{2}{*}{ Decisión } \\
\hline & CICT & CIST & SIST & CICT & CIST & SIST & \\
\hline ITPM & 0,06 & 0,44 & 0,15 & 0,00 & 0,00 & 0,00 & $\mathrm{I}(1)$ \\
\hline \multicolumn{8}{|c|}{ Tasas de interés pasivas } \\
\hline TP_SF & 0,08 & 0,49 & 0,08 & 0,00 & 0,00 & 0,00 & $\mathrm{I}(1)$ \\
\hline TBP & 0,09 & 0,56 & 0,15 & 0,00 & 0,00 & 0,00 & $\mathrm{I}(1)$ \\
\hline TD6_BE & 0,16 & 0,43 & 0,19 & 0,00 & 0,00 & 0,00 & $\mathrm{I}(1)$ \\
\hline TD6_BP & 0,04 & 0,39 & 0,13 & 0,00 & 0,00 & 0,00 & $\mathrm{I}(1)$ \\
\hline \multicolumn{8}{|c|}{ Tasas de interés activas } \\
\hline TA_SF & 0,14 & 0,38 & 0,13 & 0,00 & 0,00 & 0,00 & $\mathrm{I}(1)$ \\
\hline TOA_BE & 0,14 & 0,59 & 0,17 & 0,00 & 0,00 & 0,00 & $\mathrm{I}(1)$ \\
\hline TOA_BP & 0,46 & 0,35 & 0,15 & 0,00 & 0,00 & 0,00 & $\mathrm{I}(1)$ \\
\hline
\end{tabular}

*CICT: con intercepto y con tendencia, CIST: con intercepto y sin tendencia, SIST: sin intercepto y sin tendencia Fuente: Elaboración propia. 
CUADRO 2

RESIDUOS DE LOS MODELOS DE LARGO PLAZO HIPÓTESIS NULA: PRESENCIA DE RAÍZ UNITARIA* PERIODO ENERO DE 2000-JULIO DE 2013

\begin{tabular}{lcc}
\hline Tasa & Probabilidad & Decisión \\
\hline TP_SF & 0,00 & $\mathrm{I}(0)$ \\
TBP & 0,00 & $\mathrm{I}(0)$ \\
TD6_BE & 0,00 & $\mathrm{I}(0)$ \\
TD6_BP & 0,00 & $\mathrm{I}(0)$ \\
TA_SF & 0,02 & $\mathrm{I}(0)$ \\
TOA_BE & 0,02 & $\mathrm{I}(0)$ \\
TOA_BP & 0,01 & $\mathrm{I}(0)$ \\
\hline
\end{tabular}

*Sin constante y sin tendencia

Fuente: Elaboración propia.

Anexo 4

MODELOS DE CORRECCIÓN DEL ERROR CON ASIMETRÍA PRUEBA DE WALD HO: PERIODO ENERO DE 2000-JULIO DE 2013

\begin{tabular}{lccc}
\hline Tasa & Estadístico t & Estadístico F & Probabilidad \\
\hline TP_SF & 0,51 & 0,26 & 0,61 \\
TBP & $-1,66$ & 2,76 & 0,10 \\
TD6_BE & $-0,51$ & 0,26 & 0,61 \\
TD6_BP & $-3,23$ & 10,41 & 0,00 \\
TA_SF & $-2,21$ & 4,88 & 0,03 \\
TOA_BE & $-0,54$ & 0,30 & 0,59 \\
TOA_BP & $-2,63$ & 6,91 & 0,01 \\
\hline
\end{tabular}

Fuente: Elaboración propia. 


\section{Anexo 5}

COEFICIENTES Y VELOCIDAD DE TRASPASO DEL ITPM A LAS TASAS DEL SISTEMA FINANCIERO MODELOS DE CORTO PLAZO: PERIODO 2000M1 2013M7

\begin{tabular}{|c|c|c|c|c|c|c|c|}
\hline \multirow{2}{*}{ Periodos } & \multicolumn{4}{|c|}{ Pasivas } & \multicolumn{3}{|c|}{ Activas } \\
\hline & TBP & TP_SF & TD6_BE & TD6_BP & TA_SF & TOA_BE & TOA_BP \\
\hline Coeficiente de traspaso de corto plazo & 0,17 & 0,13 & 0,16 & 0,15 & 0,08 & 0,07 & 0,12 \\
\hline Desv. estándar & 0,04 & 0,03 & 0,04 & 0,07 & 0,04 & 0,03 & 0,06 \\
\hline
\end{tabular}

A. Modelo con asimetría durante PA

\begin{tabular}{|c|c|c|c|c|c|c|c|}
\hline MCE para desviaciones negativas & $-0,12$ & $-0,04$ & $-0,11$ & $-0,12$ & $-0,10$ & $-0,07$ & $-0,12$ \\
\hline Desv. estándar & 0,04 & 0,03 & 0,05 & 0,04 & 0,03 & 0,03 & 0,04 \\
\hline MCE para desviaciones positivas & $-0,05$ & $-0,05$ & $-0,11$ & $-0,08$ & $-0,04$ & $-0,06$ & $-0,07$ \\
\hline Desv. estándar & 0,03 & 0,02 & 0,05 & 0,02 & 0,03 & 0,02 & 0,03 \\
\hline \multicolumn{8}{|l|}{ B. Modelo con asimetría durante BC } \\
\hline MCE para desviaciones negativas & $-0,16$ & $-0,18$ & $-0,11$ & $-0,20$ & $-0,16$ & $-0,12$ & $-0,18$ \\
\hline Desv. estándar & 0,04 & 0,04 & 0,04 & 0,06 & 0,03 & 0,04 & 0,03 \\
\hline MCE para desviaciones positivas & $-0,09$ & $-0,16$ & $-0,08$ & $-0,02$ & $-0,07$ & $-0,10$ & $-0,07$ \\
\hline Desv. estándar & 0,03 & 0,03 & 0,03 & 0,02 & 0,03 & 0,03 & 0,03 \\
\hline
\end{tabular}

C. Velocidad de ajuste en meses

\begin{tabular}{lrrrrrrr} 
Modelo general & 8 & 10 & 8 & 10 & 11 & 12 & 8 \\
Ante choques negativos durante PA & 6 & 21 & 7 & 6 & 8 & 12 & 6 \\
Ante choques positivos durante PA & 14 & 16 & 7 & 9 & 21 & 14 & 11 \\
Ante choques negativos durante BC & 5 & 5 & 7 & 4 & 6 & 7 & 5 \\
Ante choques positivos durante BC & 9 & 6 & 10 & 44 & 12 & 9 & 12 \\
\hline
\end{tabular}

Nota: los valores en rojo se calcularon a partir de valores que resultaron no ser significativos al $10 \%$.

Fuente: Elaboración propia. 


\section{Anexo 6}

EVOLUCIÓN DEL ÍNDICE DE CONCENTRACIÓN BANCARIA DE ACTIVOS HERFINDAHL-HIRSCHMAN (H-H) ENERO 1999 - JULIO 2013

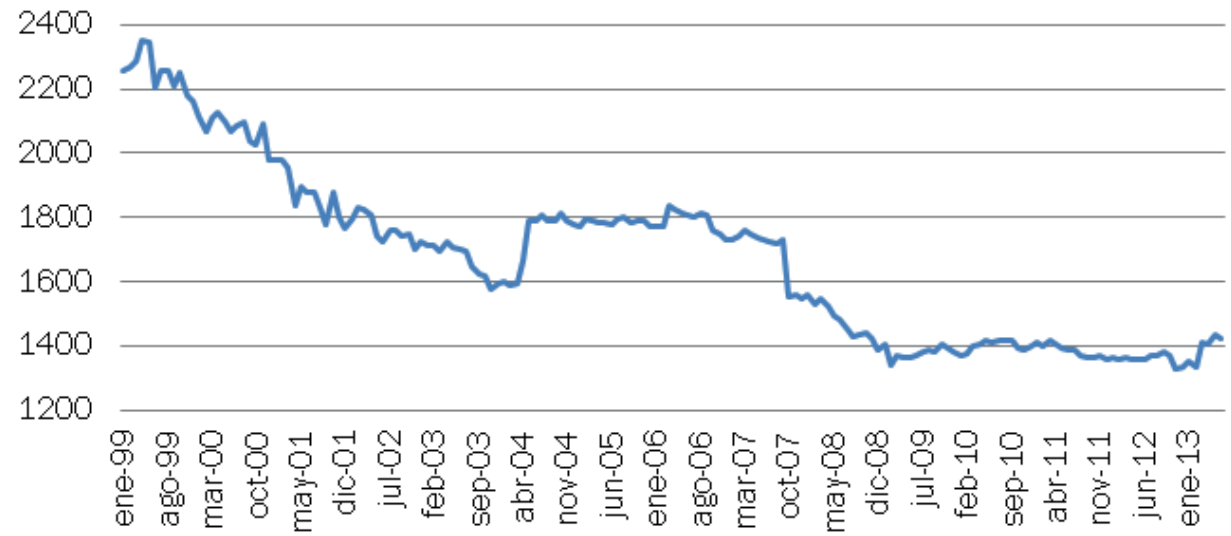

Fuente: Elaboración propia.

\section{Anexo 7}

PRUEBA DE IGUALDAD DE COEFICIENTES

N. ${ }^{\circ}$ DE OBS: 163

\begin{tabular}{lc}
\hline & Hipótesis nula: A-B=0 \\
\hline A. Coeficiente TA_SF & $-0,068$ \\
B. Coeficiente TP_SF & $-0,059$ \\
Desviación estándar común & 0,002 \\
Estadístico t & 4,57 \\
Probabilidad & Hipótesis nula: C-D=0 \\
\hline & \\
\hline C. Coeficiente TD6_BE & 0,00 \\
D. Coeficiente TP_SF & $-0,080$ \\
Desviación estándar común & $-0,097$ \\
Estadístico t & 0,002 \\
Probabilidad & Hipótesis nula: E-F=0 \\
\hline & 9,29 \\
\hline E. Coeficiente TOA_BE & 0.00 \\
F. Coeficiente TOA_BP & \\
Desviación estándar común & $-0,093$ \\
Estadístico t & $-0,041$ \\
Probabilidad & 0,002 \\
\hline
\end{tabular}

Fuente: Elaboración propia. 


\section{Anexo 8}

HIPÓTESIS NULA: $\Gamma_{131}=\Gamma_{132}$

\begin{tabular}{lcccc}
\hline Var/Prueba & Estadístico $t$ & Probabilidad & Estadístico F & Probabilidad \\
\hline TBP & $-0,91$ & 0,36 & 0,83 & 0,36 \\
TP_SF & $-1,16$ & 0,25 & 1,35 & 0,25 \\
TD6_BE & $-0,94$ & 0,35 & 0,88 & 0,35 \\
TD6_BP & $-1,07$ & 0,28 & 1,16 & 0,28 \\
TA_SF & $-0,53$ & 0,59 & 0,29 & 0,59 \\
TOA_BE & 0,32 & 0,75 & 0,10 & 0,75 \\
TOA_BP & $-2,56$ & 0,01 & 6,55 & 0,01 \\
\hline
\end{tabular}

Fuente: Elaboración propia. 
